\section{CASE REPORT}

P. de Graaf

P. van der Valk

A.C. Moll

S.M. Imhof

A.Y.N. Schouten-van

Meeteren

J.A. Castelijns

\title{
Retinal Dysplasia Mimicking Intraocular Tumor: MR Imaging Findings with Histopathologic Correlation
}

SUMMARY: We report a 6-month-old boy who presented with unilateral leukocoria, retinal detachment, and a retrolental mass in a microphthalmic eye based on retinal dysplasia with concurrent optic nerve aplasia. Dysplastic retinal tissue, a rare congenital defect, may create a clinical and radiologic picture of an intraocular mass closely resembling tumor tissue. MR imaging findings with histopathologic correlation are presented to facilitate discrimination of the more common causes of leukocoria.

$\mathbf{R}$ etinal dysplasia is a rare cause of childhood leukocoria, which can cause considerable diagnostic difficulty in the differentiation of benign and malignant intraocular pathology. When clinical diagnosis is uncertain, ocular MR imaging helps to characterize and differentiate between intraocular pathologies. However, in contrast to many lesions in the differential diagnosis of leukocoria, to our knowledge, detailed MR imaging findings in retinal dysplasia have not been previously reported. We report MR imaging findings of unilateral retinal dysplasia with concurrent optic nerve aplasia.

\section{Case Report}

A 6-month-old boy was admitted with a history of a smaller left eye since birth and leukocoria for 1 month. He was born to nonconsanguineous parents at 41 weeks' gestation. Ophthalmic examination revealed no abnormalities in the right eye. The left eye was microphthalmic, with a corneal diameter of $9 \mathrm{~mm}$ (right, $10 \mathrm{~mm}$ ). A yellowish-white retrolental mass, projecting from the inferomedial quadrant into the vitreous, was present (Fig 1). The mass in combination with retinal detachment obscured clear visualization of optic nerve disc and macula. Prominent irregular feeder vessels and small focal hemorrhages covered the surface of the mass. Differential diagnosis based on these findings included ciliary body medulloepithelioma, anteriorly located retinoblastoma, and persistent hyperplastic primary vitreous (PHPV).

Ultrasonography showed a noncalcified mass with high reflectivity. MR imaging showed a mass arising from the nasal ciliary body region with high signal intensity (SI) on T1-weighted (T1WI) and low SI on T2-weighted (T2WI) images, combined with a normal-appearing vitreous and tent-shaped retinal detachment with subretinal exu-

Received November 21, 2006; accepted after revision April 3, 2007.

From the Departments of Radiology (P.d.G., J.A.C.), Pathology (P.v.d.V.), Ophthalmology (A.C.M.. S.M.I.), and Pediatric Oncology (A.Y.N.S.v.M.), VU University Medical Center, Amsterdam, the Netherlands; and the Department of Pediatric Oncology (A.Y.N.S.v.M.), Emma Children's Hospital, Academic Medical Center, Amsterdam, the Netherlands.

P.d.G. is financially supported in part by grants from ZonMw (Netherlands Organization for Health Research and Development) 's-Gravenhage, the Netherlands; the ODAS Foundation, Delft, The Netherlands; the National Foundation for the Blind and Visually Impaired, Utrecht, the Netherlands; the Blindenhulp Foundation 's-Gravenhage, the Netherlands; and the Dutch Eye Fund, Utrecht, the Netherlands.

Please address correspondence to Pim de Graaf, MD, Department of Radiology, VU University Medical Center, De Boelelaan 1117, 1081 HV Amsterdam; e-mail: p.degraaf@vumc.nl

DOI 10.3174/ajnr.A0635

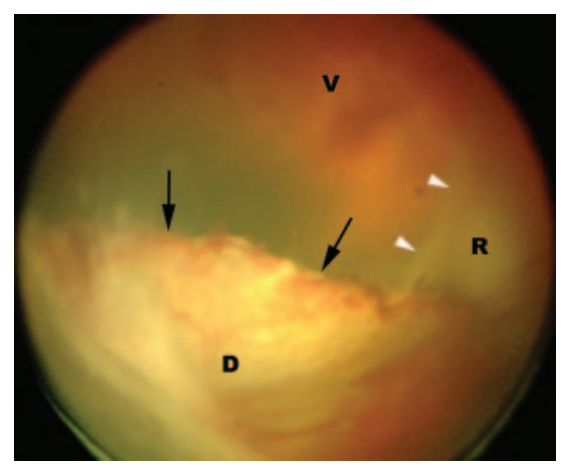

Fig 1. Fundus photograph of the left eye shows a retrolental mass (D, arrows) in the inferomedial quadrant of the vitreous (V) with large irregular feeder vessels, focal hemorrhages, and retinal detachment $(\mathrm{R}$, arrowheads)

date (Fig 2). The anterior chamber and lens showed no abnormalities. The right orbit, optic nerve, and portions of the optic chiasm related to the nerve were normal. The optic nerve and sheath complex were not visible within the left orbit or anywhere along the usual course of the nerve (Fig 2). Total aplasia of the left optic nerve was not combined with other congenital central nervous system (CNS) abnormalities. The mass showed intense homogeneous enhancement. Ultrasonography and MR imaging findings were more consistent with medulloepithelioma or PHPV, and a retinoblastoma could be ruled out. Five weeks later, enucleation was required due to enlargement of the mass with an increase of intraocular pressure, shallowness of the anterior chamber, and progression toward a phthisis bulbi.

Histopathologic examination revealed a total absence of the optic nerve, scleral lamina cribrosa, and central retinal vessels. A vascularized mass was present anteriorly in the vitreous cavity on either side of the lens, adjacent to the ciliary body (Fig 3). The anterior eye segment showed no abnormalities. The mass consisted of an abnormal retinal differentiation, with disorganization, proliferation, and rosette formation in the photoreceptor cell layer. In the thin retina that was not incorporated in the mass, no ganglion cell layer was present. The posterior eye segment showed no persisting hyaloid artery nor a thin stalk connecting the posterior lens and the optic disc region, presumed to represent a remnant of the Cloquet canal. The pathologic diagnosis was retinal dysplasia with concurrent optic nerve aplasia.

\section{Discussion}

Retinal dysplasia is more of a descriptive term, rather than a delineation of a clinical condition and may be a component of 

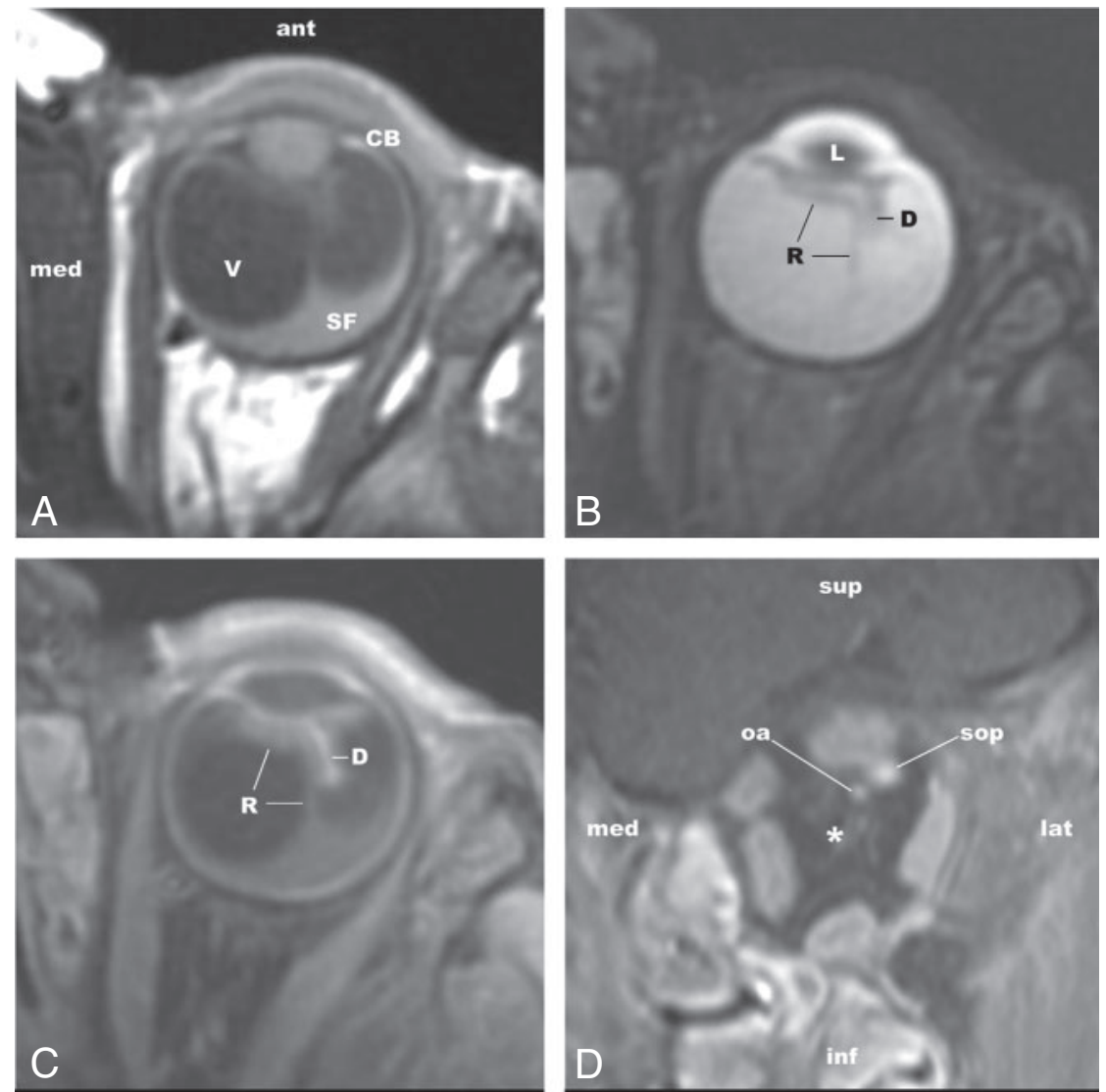

a group of congenital disorders, such as Norrie disease, trisomy 13, or Walker-Warburg syndrome. Ocular findings in these disorders are mostly bilateral and accompanied by systemic and CNS abnormalities. Unilateral retinal dysplasia is usually not associated with a specific syndrome. Histopathologic examination demonstrates a reduction or complete absence of retinal ganglion cells, their nerve fibers, and retinal blood vessels, which can result in a congenital (usually tentshaped) retinal detachment, a retinal malformation combined with rosette formation and optic nerve aplasia.

Clinically, retinal dysplasia may present itself in a wide range of severity, from simple to massive folding of the retina, ical-appearing tumor, MR imaging
Fig 2. Axial T1WI (A) image shows a hyperintense mass in the anterior part of the vitreous (V), adjacent to the ciliary body (CB) on either side of the lens, combined with a tent-shaped retinal detachment with hyperintense subretinal fluid (SF). T2WI (B) image shows retinal detachment as a hypointense fine linear structure (R) and clearly demarcates the dysplastic retinal tissue (D). After we applied the contrast material $(C$, contrast-enhanced fat-suppressed TIWI image), the latter shows enhancement (D), in contrast to the detached retina (R) and subretinal fluid. Coronal contrastenhanced fat-suppressed T1WI $(D)$ MR images reveal absence of the optic nerve (asterisk) in an otherwise normal orbit. sop indicates superior ophthalmic vein; oa, ophthalmic artery.

central stalks with retinal tissue extending from the optic disc to the back of the lens, and complete retinal detachment leading to highly disorganized microphthalmic eyes. ${ }^{1}$ If the intraocular proliferation is large enough, it may produce leukocoria. Leukocoria and retinal detachment in children require prompt further investigation because retinoblastoma should be included in the differential diagnosis. Usually, the underlying disease causing leukocoria can be deducted by ophthalmoscopy alone or in combination with ultrasonography. When clinical diagnosis is uncertain, caused by an obscured view or an atyphelps to characterize and differentiate between intraocular pathologies.

On MR imaging, a retinal detachment presents as a fine linear structure, which is limited anteriorly at the ora serrata and posteriorly at the optic disc. Compared with the vitreous, the SI of the detached retina is high on T1WI images, low on T2WI images, and might show some contrast enhancement. In our patient, retinal dysplasia showed the same SI characteristics as those of normal detached retinal tissue; however, the enhancement after gadolinium administration was much stronger. It is likely that enhancement of dysplastic retinal tissue varies with the quality and number of blood vessels. De-
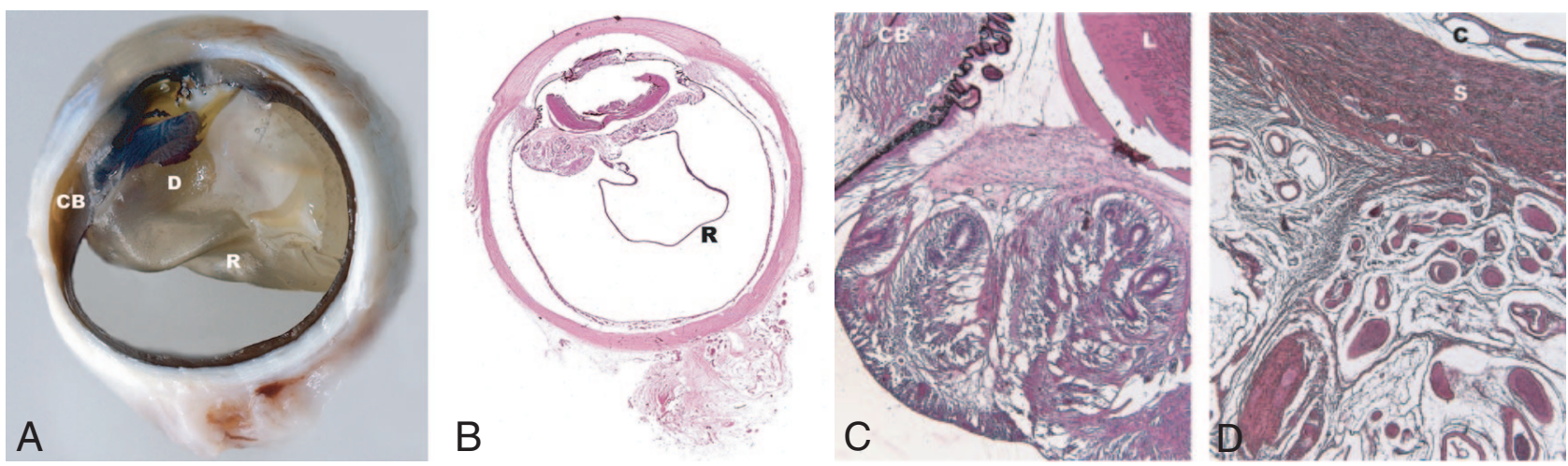

Fig 3. $A$, Gross examination shows the detached retina (R) and the yellowish dysplastic retinal tissue (D), which is adjacent to the ciliary body (CB). The subretinal fluid is located inside the tent-shaped retinal detachment (R) and cannot be seen on this gross specimen. $B$, Microscopic examination shows the dysplastic retinal tissue behind the lens arising from the retina (R). The mass does not invade the ciliary body or the anterior chamber (hematoxylin-eosin [H\&E], original magnification $\times 3.5$ objective). $C$, Detail of the mass shows still vaguely recognizable retinal tissue near the lens (L) and ciliary body (CB), with rosette formation composed of photoreceptor cells (H\&E, original magnification $\times 20$ objective). $D$, Detail of the region in which the optic nerve was expected shows absence of the optic disc, lamina cribrosa, and optic nerve. Orbital connective tissue with a collection of small (ciliary) vessels is present behind the eye (H\&E, original magnification $\times 20$ objective). $C$ indicates choroid; $S$, sclera. 
spite thickening of the dysplastic retinal tissue, the characteristic linear structure of a retinal detachment was preserved. This MR imaging feature might allow differentiation of retinal dysplasia from lesions, such as retinoblastoma or medulloepithelioma, which appear as solid masses.

The differential diagnosis of a retrolental intraocular mass in children, with a hyperintense SI on T1WI and a hypointense SI on T2WI images, combined with retinal detachment, which can have MR imaging features identical to those in our patient, can be anteriorly located retinoblastoma, PHPV, or medulloepithelioma. Anteriorly located retinoblastoma can present without characteristic calcifications and is more common in older children compared with typical cases; it arises from the posterior retina in younger children., ${ }^{2,3}$ Theoretically, positron-emission tomography might differentiate dysplasia and neoplasms, but this differentiation has not been confirmed for retinoblastoma. ${ }^{4}$ However, findings of vitreous seeding or extension into the iris and anterior chamber may help to diagnose retinoblastoma.

PHPV results from failure of embryonic fibrovascular primitive stroma (the hyaloid system) to regress; it may continue to proliferate and create a tubular structure extending from the posterior surface of the lens to the optic disc. ${ }^{5}$ This solid component usually shows enhancement on postcontrast images. The common presence of a retinal detachment, ab- normalities of the lens and anterior chamber, and absence of calcifications can be helpful in the diagnosis.

Masses that are localized mainly in the ciliary body are more suggestive of medulloepithelioma, a congenital tumor of the ciliary body epithelium. From the ciliary body, the tumor may spread posteriorly along the retina and anteriorly along the posterior surface of the lens. Calcifications and retinal detachment are associated findings seen in (advanced) medulloepithelioma, and the tumors are well enhanced after contrast administration.

Although the diagnosis of intraocular pathology is usually clinically obvious and the presented MR imaging findings are not specific for retinal dysplasia, awareness of this disease and the presentation on MR imaging might be helpful to differentiate between other benign or malignant intraocular pathology and to reach an early diagnosis.

\section{References}

1. Godel V, Nemet P, Lazar M. Retinal dysplasia. Doc Ophthalmol 1981;51:277-88

2. Karcioglu ZA, Abboud EB, Al Mesfer SA, et al. Retinoblastoma in older children. J AAPOS 2002;6:26-32

3. Vajaranant TS, Mafee MF, Kapur R, et al. Medulloepithelioma of the ciliary body and optic nerve: clinicopathologic, CT, and MR imaging features. $\mathrm{Neu}$ roimaging Clin N Am 2005; 15:69-83

4. Moll AC, Hoekstra OS, Imhof SM, et al. Fluorine-18 fluorodeoxyglucose positron emission tomography (PET) to detect vital retinoblastoma in the eye: preliminary experience. Ophthalmic Genet 2004;25:31-35

5. Castillo M, Wallace DK, Mukherji SK. Persistent hyperplastic primary vitreous involving the anterior eye. AJNR Am J Neuroradiol 1997;18:1526-28 